\title{
Study of temporal correlations in the urban noise monitoring network of Milan, Italy
}

\author{
Roberto Benocci $^{1}$ H. Eduardo Roman ${ }^{2}$ Chiara Confalonieri ${ }^{1} \quad$ Giovanni Zambon $^{1}$ \\ ${ }^{1}$ Department of Environmantal Sciences, University of Milano Bicocca, 20126 Milano, Italy \\ ${ }^{2}$ Department of Physics, University of Milano Bicocca, 20126 Milano, Italy
}

Received: July 2, 2020. Revised: August 25, 2020. Accepted: September 2, 2020. Published: September 4, 2020.

\begin{abstract}
The European Life project, called DYNAMAP, has been devoted to provide a real image of the noise generated by vehicular traffic in urban and suburban areas, developing a dynamic acoustic map based on a limited number of low-cost permanent noise monitoring stations. In the urban area of Milan, the system has been implemented over the pilot area named Area 9. Traffic noise data, collected by the monitoring stations, each one representative of a number of roads with similar characteristics (e.g. daily traffic flow), are used to build-up a "real time" noise map. DYNAMAP has a statistical structure and this implies that information captured by each sensor must be representative of an extended area, thus uncorrelated from other stations. The study of the correlations among the sensors represents a key-point in designing the monitoring network. Another important aspect regards the "contemporaneity" of noise fluctuations predicted by DYNAMAP with those effectively measured at an arbitrary location. Integration times heavily affect the result, with correlation coefficients up to 0.8-0.9 for updating times of $1 \mathrm{~h}$. Higher correlations are observed when averaging over groups of roads with similar traffic flow characteristics.
\end{abstract}

Keywords- DYNAMAP, Dynamic noise map, Noise prediction, Temporal correlations.

\section{INTRODUCTION}

$\mathbf{N}^{\circ}$ OISE mapping are becoming a necessary tool for evaluating the noise exposure of citizens in large cities, as it has been recognized by the strict doseharmful effect relationships reported both in the Directive 2002/49/EC 1] and the 2018 WHO Environmental noise guidelines [2].

Strategic Noise Maps have been implemented to enable effective diagnostics on the acoustic environment and provide useful information for local intervention measures and policy-making [3, 4]. They evaluate the overall exposure to noise in a given area due to different sources and, together with Action Plans, provide a framework to manage environmental noise and its effects. Thus, they represent the usual approach for noise prevention and control [5, 6. Recently, noise maps have been evolving towards a multi-source predictive approach [7, 8, 9, 10] also implemented by information regarding vehicles speed [11, 12] and annoyance [13, 14. However, the introduction of dynamic noise maps constituted a further evolution in the direction of better representing the "real" noise exposure.

To this end, the European project DYNAMAP 15 has developed a dynamical acoustic map in two pilot areas: a large portion of the urban area of the city of Milan (Area 9) 16. and the motorway surrounding Rome [17. In both cases, one can predict traffic noise in an extended area using a limited number of monitoring sensors and the knowledge of traffic flows. Traffic noise data, collected by the monitoring stations, each one representative of a number of roads within the pilot area showing similar characteristics (e.g. daily traffic flow), are used to build-up a "real time" noise map [18, 10, 20].

DYNAMAP relies on the update of pre-calculated basic noise maps. This process, denoted as noise map scaling, is accomplished for different operating conditions (sources, traffic and weather conditions), by detecting noise and meteorological data from low-cost monitoring stations and weather sensors distributed along the road (just for Rome's pilot area). The scaled basic noise maps, one for each elementary noise source present in the mapping area, are summed together to obtain the complete noise map. This process is expected to considerably reduce the use of simulation tools, thus decreasing calculation times and costs. In addition, the development of low cost noise monitoring stations and the use of a GIS platform for implementing the maps scaling and their energetic sum, further reduces operational costs. DYNAMAP assumes that the noise patterns of each selected group of raods to be stationary. The noncompliance with this assumption would introduce a bias in the system which would need groups reassignment.

In a more general perspective, several issues related to the ones considered here have been discussed recently, 
such as the use of green walls in cities [21, new vehicle speed models for residential areas [22], and the impact of ring roads on a surrounded city 23 .

In this work, we are interested in studying how traffic noise in different parts of the city is correlated. This knowledge is important for a better design of the network, so that the sensors cover the pilot zone in an optimized way. First, we study the correlations among the 24 monitoring units building up the DYNAMAP network, and second, we analize the "contemporaneity" of noise fluctuations predicted by DYNAMAP and compare them with field measurements at arbitrary locations in the pilot zone.

The paper is organized as follows. In Section II. we briefly review the DYNAMP sensor network concepts and main strategy. In Section III., we study the noise correlations among sensors, and the corresponding DYNAMAP predictions. Section IV. is devoted to the Discussion of the results, and Sect. V. to the Conclusions.

\section{NETWORK OF SENSORS AND DYNAMIC NOISE MAPPING}

A sample made of 93 noise time series (24-hour each), distributed over the entire city of Milan, has been analyzed by standard clustering techniques. The result of the analysis is shown in Fig. 1 illustrating the two mean normalized noise cluster profiles, $\bar{\Delta}_{k}, k=1,2$.

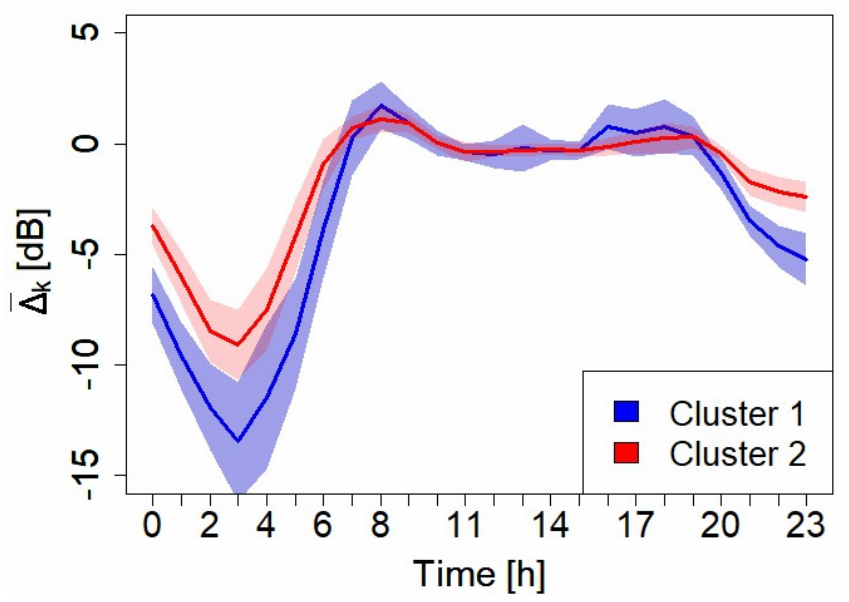

Fig. 1: Mean normalized cluster profiles, $\overline{\Delta_{k}}$, and the corresponding \pm standard deviations. Here, $k=1,2$ indicates the cluster index.

The two clusters present a similar trend during the daytime but behave differently during the evening/nighttime and the morning rush-hour. This result suggested the idea of describing the noise profile of an arbitrary road as a combination of the two mean cluster profiles 24, 25, 26. The traffic noise at any road section can be predicted if knowledge of a non-acoustic parameter $x$ is available for each road belonging within the entire road network. In our case $x$ represents the logarithm of the total daily traffic flow, $T$, of a single road [27, 28, 29. For instance if say, $T=3000$ vehicles/day, then $x=3.5$.
Since our aim is to use a small number of sensors, we need to aggregate the roads into groups showing a similar traffic behaviour. Thus, we divided the entire range of $x$ values into groups in such a way that each group contains approximatly the same number of roads.

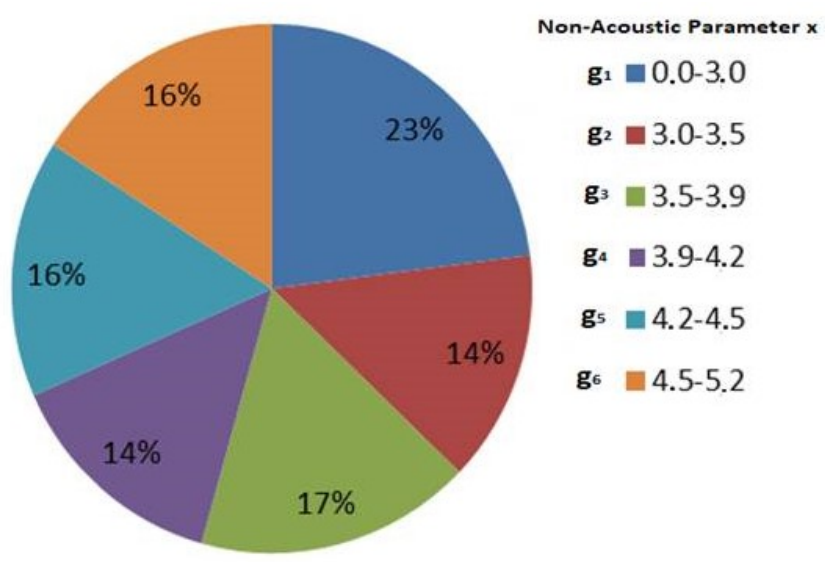

Fig. 2: Percentage of roads in each group. The intervals of variability of the non-acoustic parameter $x=\log T$ are also shown for each group $\left(g_{1}, g_{2}, \ldots, g_{6}\right)$. The latter have an associated value given by the mean $\langle x\rangle$ within the group. For instance, for $g_{5}$ we have $\langle x\rangle=4.35$.

The roads are sorted into six groups according to the value of $x$ and denoted as $\left(g_{1}, g_{2}, \ldots, g_{6}\right)$ (Fig. 2] [18, 30]. For the effective implementation of DYNAMAP, we have 24 noise monitoring stations ( 4 for each group) deployed within the pilot area. Each group of roads is represented by a single noise map. This means that all the roads belonging to a group will be described by the same acoustic map. The latter is the result of two contributions:

(a) A reference static contribution (basic noise map), $L e q_{r e f\left(g_{i}\right)}\left(T_{\text {ref }}\right)$, derived from the CadnaA software at the time interval $T_{\text {ref }}=$ (08:00-09:00), for each group $g_{i}$ (Fig. 3), from which one can obtain the total static noise map (Fig. 4);

(b) A dynamic contribution $\delta\left(g_{i}\right)$ for each group $g_{i}$ retrieved from the 24 monitoring stations [31, 32 .

The level $L e q^{a}(t)$, at location $a$ at time $t$, can then be obtained by energetically adding the local contribution of each basic map, $L e q_{r e f\left(g_{i}, a\right)}$, and the dynamical variation $\delta\left(g_{i}\right)$, according to

$$
\operatorname{Leq}^{a}(t)=10 \cdot \log \sum_{i=1}^{6} 10^{\left(\operatorname{Leq}_{r e f\left(g_{i}, a\right)}+\delta\left(g_{i}\right)\right) / 10},
$$

where $\delta\left(g_{i}\right)$ is obtained by averaging the sensors' $\delta\left(g_{i, j}\right)$ in each group [16]. Here, $\delta\left(g_{i, j}\right)$ for the generic monitoring sensor $j=1, \ldots, 24$, is obtained as

$$
\delta\left(g_{i, j}\right)(t)=\operatorname{Leq}_{\left(g_{i}, j\right)}(t)-\operatorname{Leq}_{\operatorname{ref}\left(g_{i}, j\right)}\left(T_{\text {ref }}\right),
$$

where $t$ represents the updating time of the noise map also referred to as integration time. 

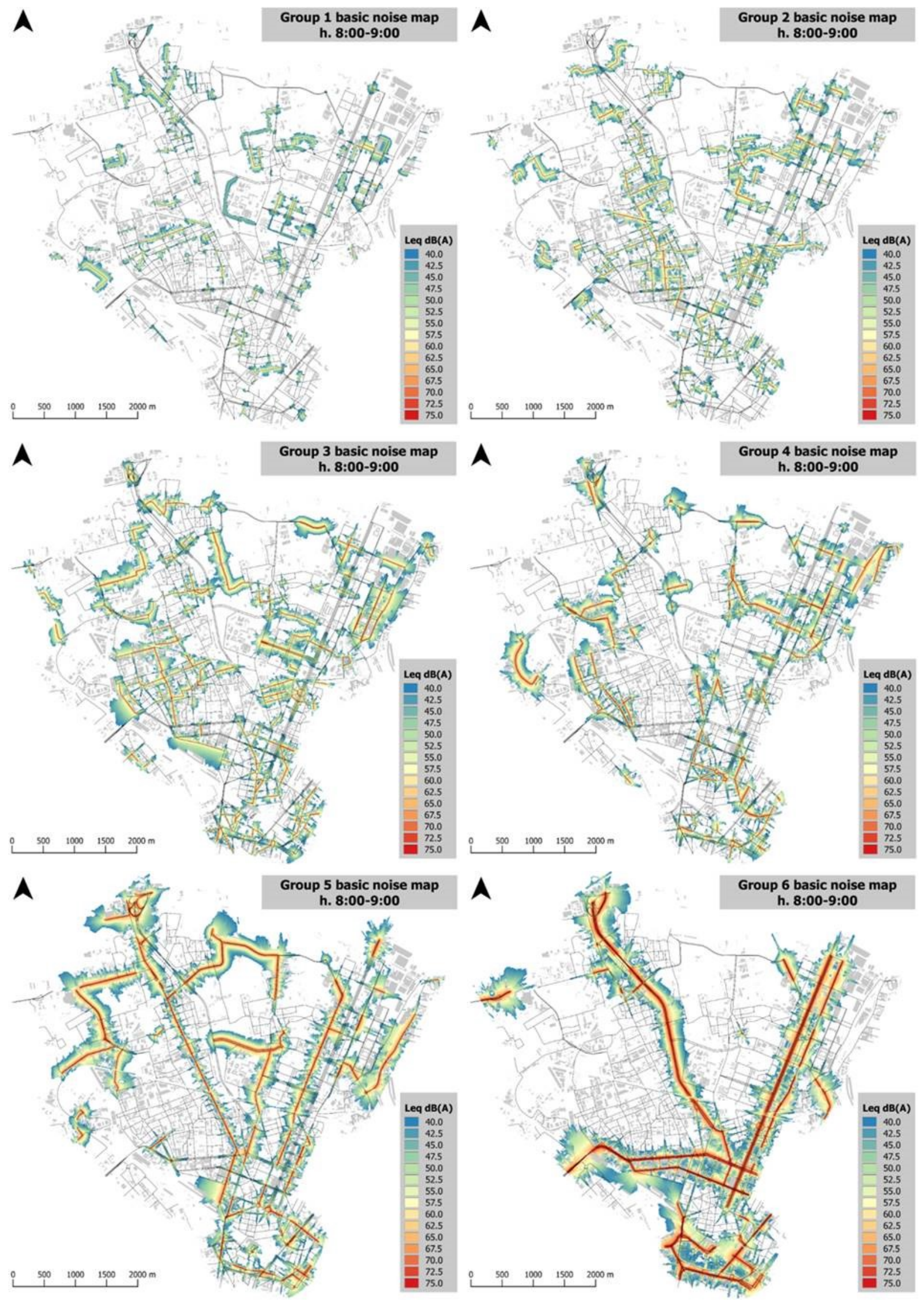

Fig. 3: Static map (basic noise map), $L e q_{r e f\left(g_{i}\right)}$, for all roads belonging to groups $g_{i}, i=1,2, \ldots ., 6$. 


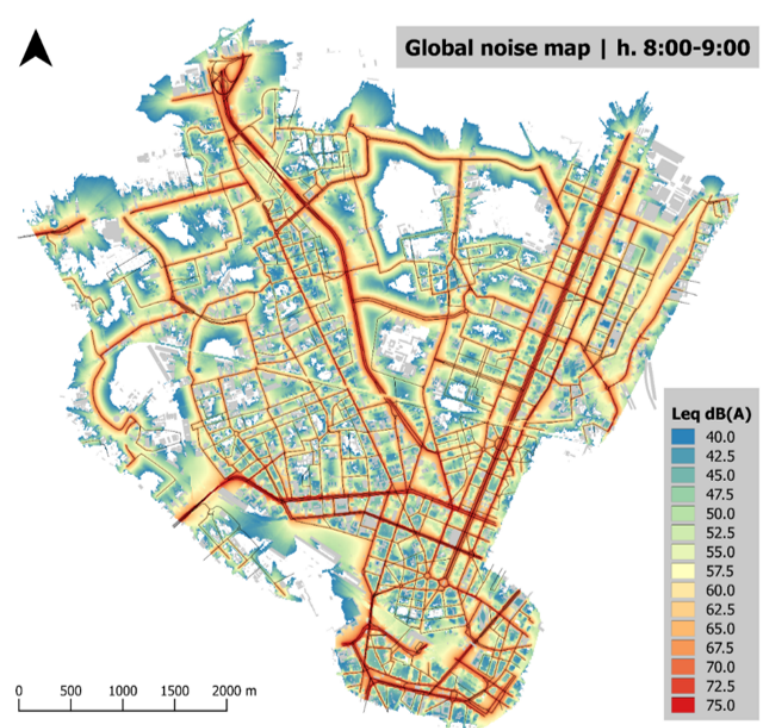

Fig. 4: Static map (basic noise map) for all roads belonging to urban Area 9.

\section{NOISE CORRELATIONS}

In this section, we study the contemporaneity of noise fluctuations as recorded by the monitoring stations, $\delta\left(g_{i, j}\right)(t)$, where $i=1, . ., 6$ and $j=1, \ldots ., 24$. Thus, we can study intragroup correlations (same index $i$ ) or intergroup ones (different $i$ ). In order to describe the temporal correlation between two sensors say, $s(i, j)$ and $s^{\prime}\left(i^{\prime}, j^{\prime}\right)$, we use the Pearson's correlation coefficient, $\rho\left(s, s^{\prime}\right)$, defined as the covariance, $\operatorname{cov}\left(s, s^{\prime}\right)$, of the two time series, $\delta\left(g_{s, s^{\prime}}\right)(t)$, divided by the product of their standard deviations, $\sigma_{s, s^{\prime}}$,

$$
\rho\left(s, s^{\prime}\right)=\frac{\operatorname{cov}\left(s, s^{\prime}\right)}{\sigma_{s} \sigma_{s^{\prime}}} .
$$

The covariance is a measure of the joint variability of the two times series for $s$ and $s^{\prime}$, and it is defined in the standard fashion as the mean value of the product of the deviations from their mean values 33 .

\section{A. Noise correlations among monitoring stations}

The recorded noise time series from the 24 monitoring stations were analyzed using a specifically developed detection algorithm [34, 35] in order to highlight the presence of possible anomalous noise events such as technical systems (thermal power stations or ventilation systems), construction sites, railway, and tram lines, which need to be erased in order to account just for traffic noise sources.

To study the noise correlations among the 24 sensors, we use the equivalent levels recorded over a period of five consecutive days to improve the statistics, and employ two normalization procedures:

(P1) From each time series, we remove the hourly median value. In this way, we obtain what we called a de-trended time series.

(P2) From each time series, we remove the mean Leq level calculated between (06:00)-(22:00). This normali- zation procedure retains both high and low frequency fluctuations.

Then, we calculate the correlation coefficient $\rho\left(s, s^{\prime}\right)$, Eq. (3), between the sensors time series obtained at five different integration times, i.e. $t=(5,10,15,30,60) \mathrm{min}$, and take the median value. The top panel in Fig. 5 reports the median of the correlation coefficient among all the de-trended monitoring stations (P1). The reported band corresponds to the median absolute deviation, $\mathrm{MAD}=\left|x_{i}-\tilde{X}\right|$, where $\tilde{X}=\operatorname{median}(X)$ and $X \equiv\left(x_{1}, \ldots, x_{n}\right)$ is a generic time series. The correlation coefficient is rather low, around 0.1 , for all the integration times considered. The results within each group $g_{i}$ are reported in the bottom panel of Fig. 5 .

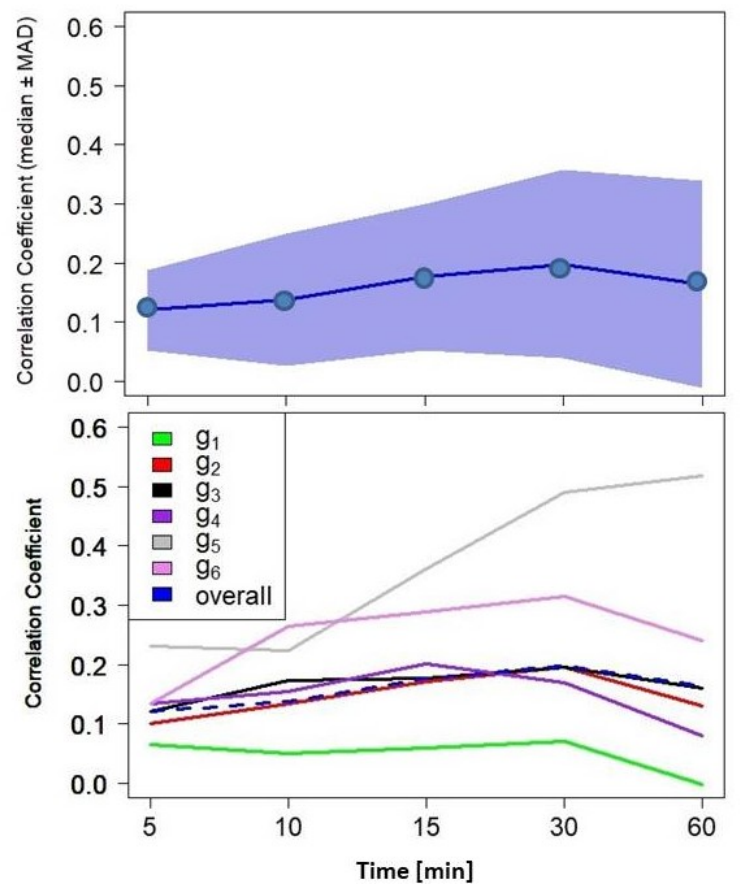

Fig. 5: Top panel: Median of the correlation coefficient among all the de-trended monitoring stations normalized according to (P1). The reported band corresponds to the median absolute deviation, MAD. Bottom panel: Same as above within each group $g_{i}(\mathrm{P} 1)$. The dashed line is the median correlation among all stations and is included for comparison.

Procedure (P1) removes all long period fluctuations, thus only high frequency fluctuations remain. To analyse this feature, we calculated the power spectrum of a fiveday time series recorded at the monitoring station $h b 129$. In this case, the normalizazion refers to a "standard" normalization, i.e. according to procedure (P2), and an example is shown in Fig. 6 for a typical 24 -h normalized (P2) noise pattern.

The resulting periodgram for the monitoring station hb129 is shown in Fig. 7. We can clearly identify two regions: a long period regime (low frequencies) and a short period regime (high frequencies). The former is associated with daily, morning and night time fluctuations (the maximum period is at $86400 \mathrm{~s}$ corresponding 


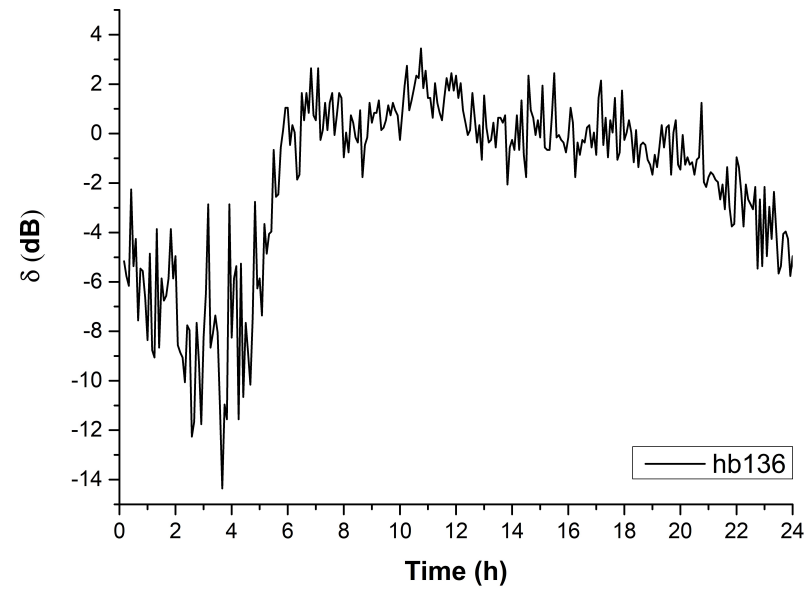

Fig. 6: Normalized Leq profile according to procedure (P2) for the monitoring station $h b 136$. Integration time: $5 \mathrm{~min}$.

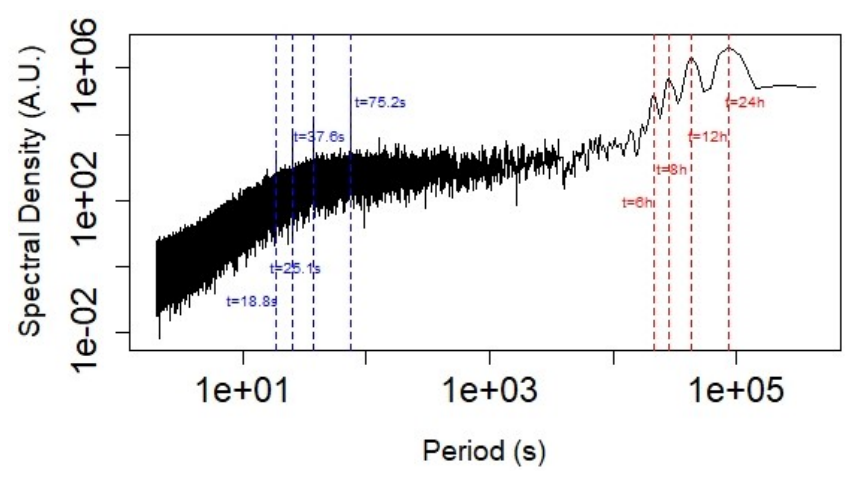

Fig. 7: Power spectrum of a five-days time series recorded by the monitoring station $h b 129$, normalized according to procedure (P2). Blue vertical lines refer to high frequency fluctuations. Red vertical lines refer to low frequency fluctuations.

to 1 day, the dashed red lines are harmonics), and the second one corresponds to time periods of the order 1 min ( $\sim 75 \mathrm{~s}$, the dashed blue lines in Fig. 7 are harmonics). The features at high frequencies are the result of short time scale fluctuations as those produced by the presence of traffic lights. Thus, the low correlation coefficients at "high frequencies" are much likely due to the unsynchronization between traffic lights which act as external driving forces. This situation is similar to what it can be observed when forced oscillations are present in an oscillating system driven by a periodic force that is external to the oscillating system. In such a case, the oscillator is compelled to move at the frequency of the driving force 36 .

From the analysis of the time series normalized according to the procedure (P2), we can observe an important increase of the correlation coefficient as we increase the updating times. At 1s integration time, the correlation coefficient is 0.4 going up to about 0.8 at $1 \mathrm{~h}$, as displayed in the top panel of Fig. 8 for all the monitoring stations. The correlation coefficients within each group are reported in the bottom panel of Fig. 8. In both bottom panels of Figs. 5 and 8 group $g_{1}$ presents the lowest correlation. This is due to the group classification process based on the road membership according to its non-acoustic parameter $x$. In fact, group $g_{1}$ contains roads with $x$ values within the interval $(0-3)$, corresponding to a total daily flow of $(1-1000)$ vehicles and therefore with a large dynamic variability. This variability impacts considerebly on the intra-group correlation over long time scales.

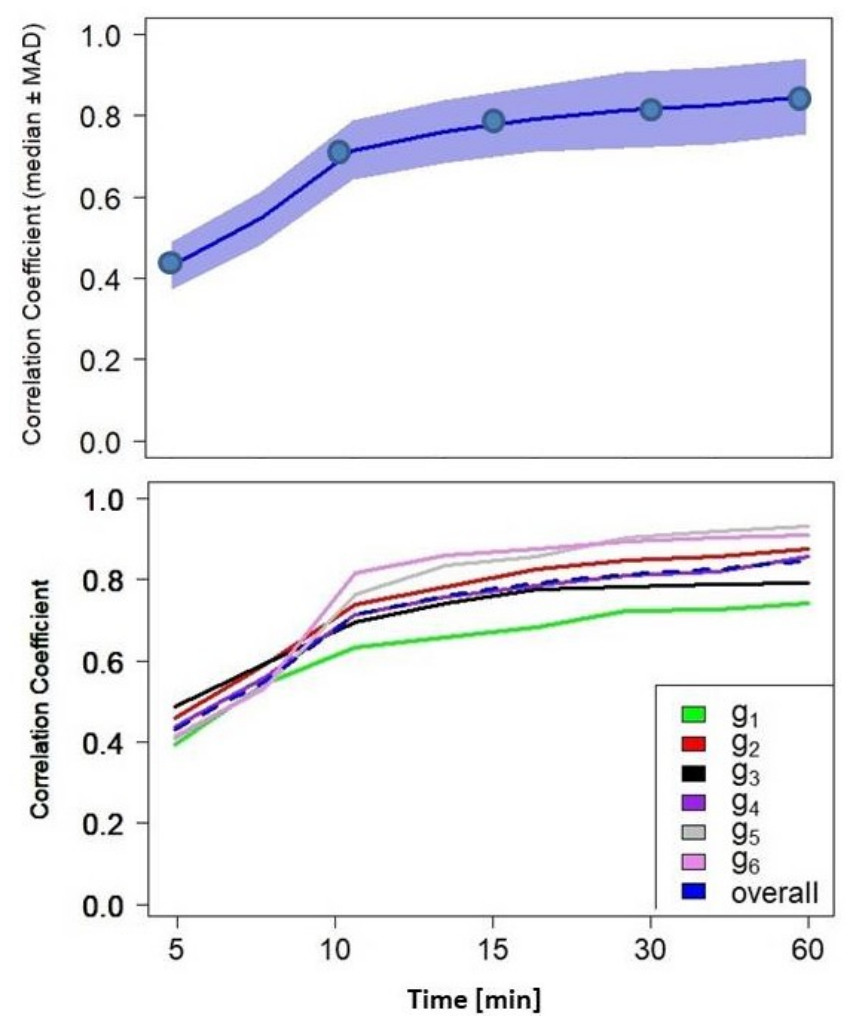

Fig. 8: Top panel: Median of the correlation coefficient among all the monitoring stations normalized according to procedure (P2). The reported band corresponds to the median absolute deviation, MAD. Bottom panel: Median of the correlation coefficients among all the monitoring stations normalized according to procedure (P2). The dashed line is the median correlation among all stations, and is included for comparison.

\section{B. Correlation of DYNAMAP predictions}

Testing the results of DYNAMAP predictions required a dedicated measurement campaign which was completed in 2019. The test measurements were performed in 21 locations within Area 9, displayed by the blue stars in Fig. 9, equally distributed in the six groups of roads. Sites were selected in order to test the system in complex scenarios where the noise from roads belonging to different groups may contribute. Figure 9 contains also the position of the 24 monitoring stations together with the indication of the six groups of roads represented by different colours. 


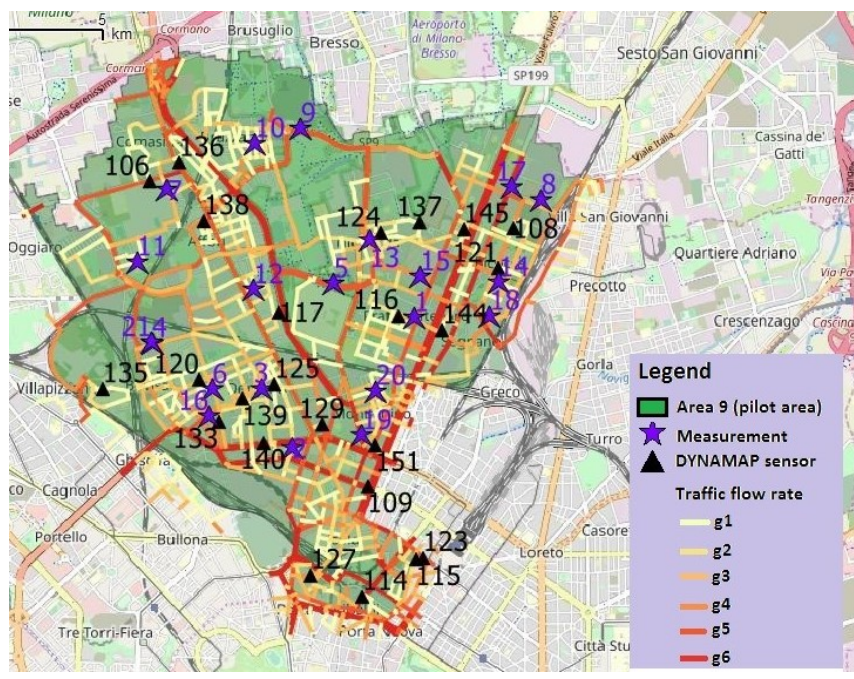

Fig. 9: Area 9 of the city of Milan. Colours correspond to the different groups of streets $\left(g_{1}, g_{2}, \ldots, g_{6}\right)$. Black triangles represent the sites where the monitoring stations are installed.

As already described in Sect. II, DYNAMAP predictions can be obtained by using Eq. 1. They are the result of the superposition of the six noise maps covering the entire Area 9.

The predefined updating times, $\tau$, within the day are: $\tau=5 \min (07: 00-21: 00) ; \tau=15 \min$ (21:00-01:00); and $\tau=60 \mathrm{~min}$ (01:00-07:00). This choice has been motivated by the need to provide the shortest time interval for the update of the acoustic maps keeping the associated error approximately constant over the entire day [19]. For the sake of simplicity, we consider, as in the previous case, an updating time constant all over the recorded period in order to highlight its influence over the correlation outcome. Figure 10 presents two different correlation results:

1) The correlation of each single measurement $\left(s_{k}, k=1, \ldots, 24\right)$ with the corresponding DYNAMAP prediction.

2) The correlation between the mean of measurements and the mean of DYNAMAP predictions $\left(g_{i}, i=\right.$ $1, \ldots, 6)$.

All the time series have been normalized according to procedure (P2). In the reported figures, the correlation coefficient increases with the integration time and presents, in some cases, quite low values at updating times of 5 and $10 \mathrm{~min}$.

As for point 2), the correlation has been calculated with the same criteria of point 1) but averaging over all the measurements and predictions in each integration interval $\tau$. In this case, the correlation results provide a higher performance even at low integration times.

\section{Discussion}

Designing a dynamic noise mapping is a complex task owing to the large number of parameters that need to be considered to describe with sufficient accuracy the traffic noise in urban areas [11. Here, we are underlying a fur- ther factor which could help reduce the redundancies of information carried by each monitoring station. When we want to describe a road network by using a statistical approach such as the one featured in DYNAMAP, we have to be sure that we are not introducing in our scheme correlated information. As the traffic flow is highly fluctuating in time on short time scales, we expect the correlation among the monitoring sensors to be very low, and actually this is the outcome illustrated in Fig. 5 with the exception of $g_{5}$. Within $g_{5}$, we observe an increase of the correlation coefficient for integration times of 30-60 min and this could be the sign of redundant information carried within the group (correlation introduced by syncronized traffic fluctuations). The other groups, instead, present low correlation coefficients and this information is useful to statistically describe the traffic source variability.

Figure 11 (top panel) illustrates the correlation matrix among the time series recorded by the monitoring network with integration time of $60 \mathrm{~min}$ and normalized according to procedure (P1). Blackish boxes indicate higher correlation which are found within $g_{5}$ ( $h b 106, h b 136, h b 151, h b 123)$. A black box appears also in correspondance with sensors $(h b 108, h b 121)$ with correlation coefficient of 0.77 . These sensors are both installed in via Pirelli, though belonging to different groups (see map in Fig. 9). Also in this case, we are using redundant information in DYNAMAP's calculation scheme. This characteristic emerges also analyzing the correlation matrix among the time series recorded by the monitoring network with integration time of $5 \mathrm{~min}$, as reported in the bottom panel of Fig. 11. In this case, the correlation coefficient between sensors $(h b 108, h b 121)$ is 0.65 . In order to better characterize these two locations, we considered the cross-correlation between these two stations. It results that the maximum correlation is achieved at a lag of $163 \mathrm{~s}$. As the distance between the two sensors is $470 \mathrm{~m}$, it means that the average traffic flow speed along via Pirelli is like $10 \mathrm{~km} / \mathrm{h}$, which is quite reasonable if we consider the presence of a traffic light in-between the two monitoring stations.

DYNAMAP requires a high correlation over long time scales, especially within each group, as groups have been assembled from similarities among noise profiles. In this sense, Fig. 8 (obtained using procedure (P2), suitable for studying both high and low frequency fluctuations) suggests that correlations are higher at longer integration times, but reduce markedly at time scales $\tau<5 \mathrm{~min}$, even within each group (see Fig. 8 bottom). This would imply that roads belonging to a group could undergo a group change as we are loosing similarities (similar noise patterns) within the groups itself.

Dynamap predictions, shown in Figs. 10, present good correlation results with the corresponding simultaneous measurements, especially at higher integration times $(\tau>10 \mathrm{~min})$. Actually, this is an expected result, as it is well known that higher updating (integration) times reduce high frequency fluctuations letting low frequency periodicities to emerge. On the other hand, 

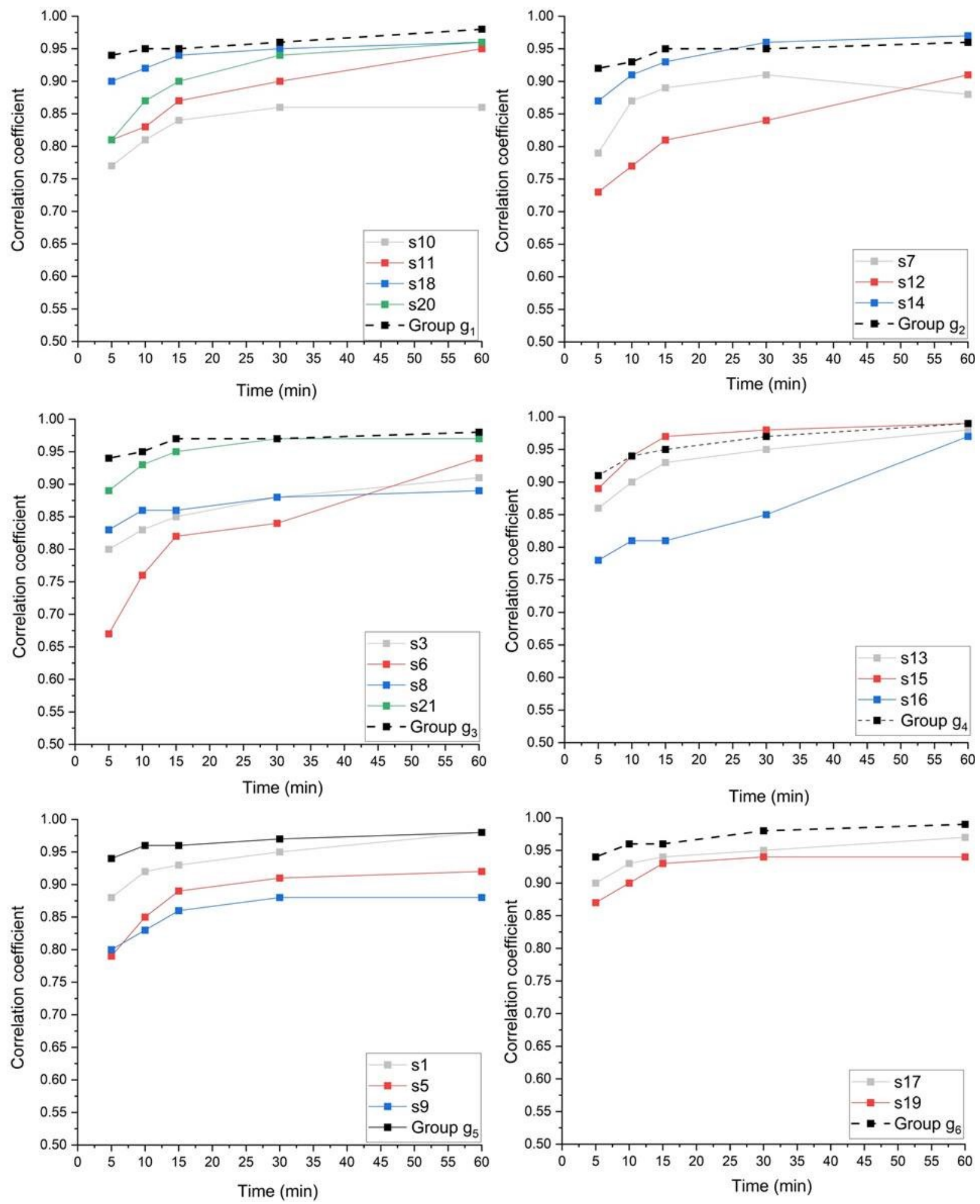

Fig. 10: Correlation coefficient between DYNAMAP predictions and field measuremets within the six groups $g_{i}$, $i=1,6$. The black dashed line illustrates the group-average correlation.

the correlation performance is enhanced at all integration times when, within each group, we calculate mean DYNAMAP's predictions and compute the correlation coefficient with the mean of the corresponding measurements. This is consistent with the fact that DYNAMAP noise description has a statistically-based approach and, therefore, its performance is enhanced when averaging over sufficient number of observations.

\section{Conclusions}

In this paper, we studied the correlations among monitoring sensors that make up the dynamic noise mapping system of DYNAMAP. The results show that the correlation coefficient the de-trended time series among 

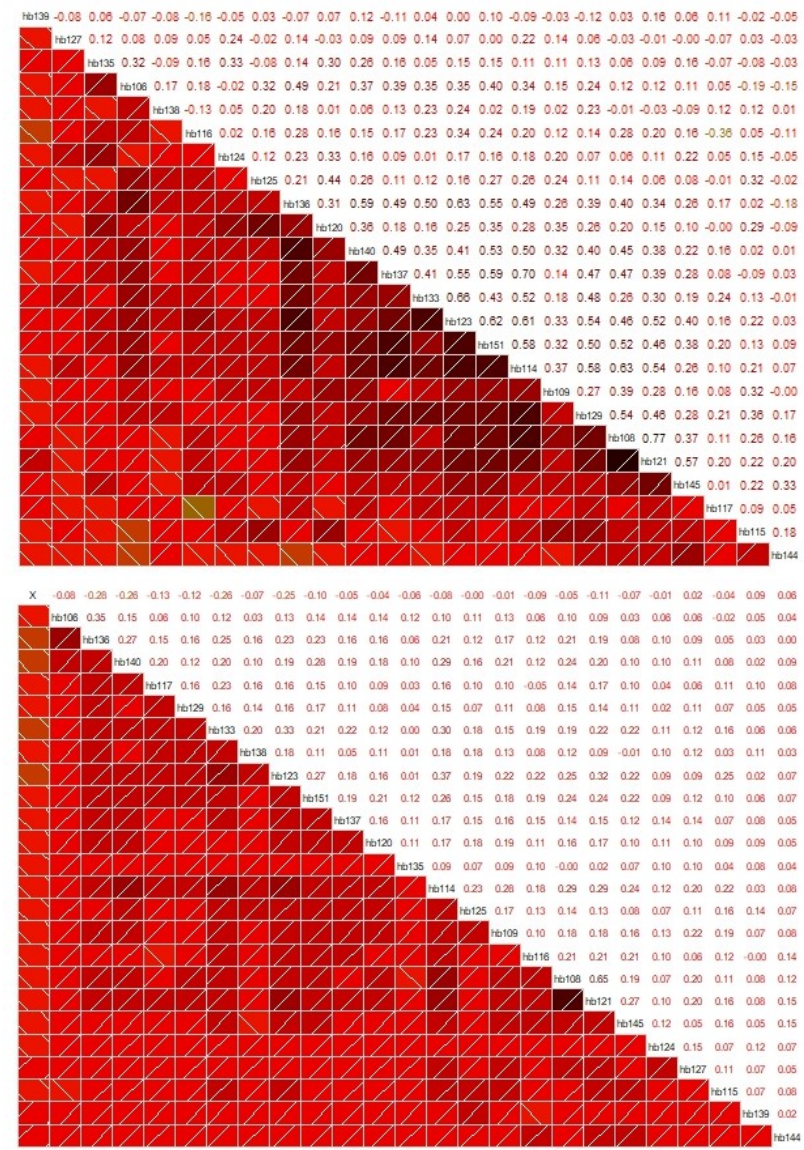

Fig. 11: Correlation matrix among the time series recorded by the monitoring network normalized according to procedure (P1). Top panel: Integration time 60 min. Bottom panel: Integration time 5 min. Blackish boxes indicate higher correlation.

all monitoring sensors, and within each group, show a very low correlation. Indeed, the presence of high frequency fluctuations associated with traffic noise shorttime scale variability makes this outcome a requirements. The exception found for $g_{5}$, for which we observe an increase of correlations over integration times 30-60 min, could be a sign that some sensor locations within the group have not been chosen appropriately. This behavior remains to be properly understood. Thus, this information is useful as it may help optimize the monitoring sensors' location and account for "distributed" noise information.

As a general rule in statistically-base noise maps, each sensor should be uncorrelated with respect to the other sensors, especially inside its own group at short-time scales (we did not go below $5 \mathrm{~min}$ ) as it represents not just the noise locally collected but also the noise from a broader area, meaning that we do not want to account for correlations due to local perturbations. Rather, we expect highly correlated behavior over long-time scales to maintain the similarities within the groups. DYNAMAP predictions are well correlated with the corresponding noise measurement (even at short integration times). As DYNAMAP has a statistically-base structure, higher correlations are observed when averaging both measurements and predictions inside each group.

Future work will be devoted to the implementation of road grid based MC simulations aimed at reproducing the observed correlations in a complex traffic network. In this way, we expect to learn how traffic noise correlations are built in and how they evolve both in time and space. The use of DYNAMAP available data will allow us to study this issue accurately and eventually draw conclusions of how to improve traffic planning in a large urban environment.

\section{REFERENCES}

[1] Directive EU. Directive 2002/49/EC of the European parliament and the Council of 25 June 2002 relating to the assessment and management of environmental noise. Off J Eur Commun L189/12 (2002).

[2] WHO Environmental noise guidelines (2018). http://www.euro.who.int/__data/assets/pdf_file/ 0008/383921/noise-guidelines-eng.pdf?ua=1.

[3] R. Klaeboe, E. Engelien, M. Steinnes, Appl. Acoust. 67 620-642 (2006).

[4] COM/2017/0151 final. European Commission 2017. Report From The Commission To The European Parliament And The Council On the Implementation of the Environmental Noise Directive in accordance with Article 11 of Directive 2002/49/EC.

[5] M. Arana, R.S. Martin, I. Nagore, D. Perez Environ. Monit. Assess., 185(6), 4951-4957 (2013).

[6] A.K. Paschalidou, P. Kassomenos, F. Chonianaki, Science of The Total Environment, 654, 144-153 (2019).

[7] G. Licitra, CRC Press (2012).

[8] J. Kang, F. Aletta, TT. Gjestland, et al., Build Environ. 108 (Supplement C): 284-94 (2016).

[9] P. Aumond, L. Jacquesson, A. Can, Applied Acoustics, 139, 34-43 (2018).

[10] C. Guarnaccia, J. Quartieri, Int. J. of Mathematical Models and Methods in Applied Sciences, 6, 926-933 (2012).

[11] G. Zambon, H.E. Roman, R. Benocci, International Journal of Environmental Research, 11(4), 449-459 (2017).

[12] G. Zambon, H.E. Roman, R. Benocci, Journal of Traffic and Transportation Engineering (English Edition), 4(3), 230-239 (2017) Open Access.

[13] G. Brambilla, C. Confalonieri, R. Benocci, Sensors 19, 23 (2019).

[14] G. Brambilla, R. Benocci, C. Confalonieri, H.E. Roman, G. Zambon, Appl. Sci., 10, 2451 (2020).

[15] DYNAMAP. 2014. Available online: http://www.life-dynamap.eu/ (accessed on 13 December 2019).

[16] R. Benocci, C. Confalonieri, H.E. Roman, F. Angelini, G. Zambon, Sensors 20, 2 (2020).

[17] R. Benocci, P. Bellucci, L. Peruzzi, A. Bisceglie, F. Angelini, C. Confalonieri, G. Zambon, Environments - MDPI 6, 79 (2019). 
[18] G. Zambon, H.E. Roman, M. Smiraglia, R. Benocci, Appl. Sci. 8(2), 251 (2018).

[19] R. Benocci, F. Angelini, M. Cambiaghi, A. Bisceglie, H.E. Roman, G. Zambon, R.M. Alsina-Pages, J.C. Socoro, F. Alias, F. Orga, INTER-NOISE 2018 47th International Congress and Exposition on Noise Control Engineering: Impact of Noise Control Engineering.

[20] R. Benocci, A. Molteni, M. Cambiaghi, F. Angelini, H.E. Roman, G. Zambon, Applied Acoustics, 156, 142-150 (2019).

[21] N. Tzortzi, J. Georgi, J. Sophocleous, WSEAS Transactions on Environment and Development 14, 270-284 (2018).

[22] N.I. Zainuddin, Y.H. Min, S.M.R. Shah, N.S.M. Sakdun, M.A. Adnan, WSEAS Transactions on Environment and Development 14, 519-525 (2018).

[23] J.A. Navas Guzman, B.R. Batista Fernandes, M. Delshad, K. Sepehrnoori, J.F. Zapata, WSEAS Transactions on Environment and Development 16, 11-22 (2020).

[24] G. Zambon, R. Benocci, A. Bisceglie, H.E. Roman. In: The 45th INTERNOISE, Hamburg, Germany (2016).

[25] X. Sevillano, J.C. Socoro, F. Alias, P. Bellucci, L. Peruzzi, S. Radaelli, P. Coppi, L. Nencini, A. Cerniglia, A. Bisceglie, R. Benocci, G. Zambon. Noise Mapping, 3, 1 (2016).

[26] G. Zambon, F. Angelini, R. Benocci, A. Bisceglie, S. Radaelli, P. Coppi, P. Bellucci, A. Giovannetti, R. Grecco, Proc. EuroNoise 2015, Maastricht, May 31 June 3 (2015).

[27] G. Zambon, R. Benocci, G. Brambilla. Int. J. Environ. Res. 10(3), 411-420 (2016).

[28] M. Smiraglia, R. Benocci, G. Zambon, H.E. Roman, Noise Mapping 3 (2016).

[29] G. Zambon, R. Benocci, G. Brambilla, Environmental monitoring and assessment 188, 26 (2016).

[30] G. Zambon, R. Benocci, A. Bisceglie, H.E. Roman, M. Smiraglia; INTER-NOISE and NOISECON Congress and Conference Proceedings, 54905496 (2017).

[31] G. Zambon, F. Angelini, M. Cambiaghi, H.E. Roman, R. Benocci, Proc. EuroNoise 2018, Crete, May 27 - 31 (2018).

[32] R. Benocci, F. Angelini, M. Cambiaghi, A. Bisceglie, H.E. Roman, G. Zambon, R.M. Alsina-Pages, J.C. Socoro, F. Alias, F. Orga, in: INTER-NOISE and NOISE-CON Congress and Conference Proceedings, 26-29 August 2018, Chicago, Illinois, USA, Institute of Noise Control Engineering.

[33] J. Cohen, 1988. Statistical power analysis for the behavioral sciences (2nd ed.). Hillsdale, NJ: Lawrence Erlbaum Associates.

[34] F. Orga, J.C. Socoro, F. Alias, R.M. Alsina-Pages, G. Zambon, R. Benocci, A. Bisceglie, 24th International Congress on Sound and Vibration, ICSV (2017).

[35] R.M. Alsina-Pages F. Alias, J.C. Socoro, F. Orga,
R. Benocci, G. Zambon, Applied Acoustics 151, 183192 (2019).

[36] A.L. Stanford, J.M. Tanner, in Physics for Students of Science and Engineering, 1985

\section{Creative Commons Attribution License 4.0 (Attribution 4.0 International, CC BY 4.0)}

This article is published under the terms of the Creative Commons Attribution License 4.0

https://creativecommons.org/licenses/by/4.0/deed.en_US 